\title{
CONTROL OF A SADDLE NODE BIFURCATION IN A POWER SYSTEM USING A PID CONTROLLER
}

\author{
D. Rosas', C. Amaro', \& J. Alvarez² \\ ${ }^{1}$ Universidad Autónoma de Baja California, Facultad de Ingeniería \\ Blvd. Benito Juárez s/n, Mexicali, B. C., México \\ E-mail: drosas@yaqui,mx.uabc.mx \\ ${ }^{2}$ Centro de Investigación Científica y de Educación Superior de Ensenada \\ Km. 107 carr. Tijuana-Ensenada, Ensenada, B.C., México \\ E-mail: jgalvar@cicese.mx \\ Received: $15^{\text {th }}$ October 2001 and accepted April $10^{\text {th }} 2002$
}

\section{ABSTRACT}

In this work, we present the elimination of a saddle-node bifurcation in a basic power system using a PID controller. In addition, a stability analysis of the rotor angle and its frequency, which are directly related to voltage collapse problem, is presented.

\section{RESUMEN}

En este trabajo se presenta la eliminación de una bifurcación de un nodo tipo "Saddle" en un sistema de potencia básica utilizando un controlador PID. Asimismo se presenta el análisis de estabilidad del ángulo del rotor y de su frecuencia que están directamente relacionadas con el problema de colapso del voltaje.

KEYWORDS: Saddle-node bifurcations, PID controller, power systems

\section{INTRODUCTION}

Voltage collapse in electric pow er systems has recently received significant attention in the literature. This has been attributed to increases in demand that result in operation of electric pow er system close to its stability limits. In several papers, for example [1, 2, 3, 4, 5], voltage collapse is related to a saddle-node bifurcation of an equilibrium point, $w$ hich occurs $w$ hen the real or reactive pow er demand is quasi-staticaly varied. Some other types of bifurcations present in a voltage collapse are Hopf, period-doubling, and cyclic bold types; furthermore, the system may display even chaotic behavior. Several previous w orks have proposed some procedures to control these bifurcations [1,7,9]; how ever, they have been focused on bifurcation control of periodic solutions.

Voltage collapse is a system instability that involves many pow er system components and their variables. This phenomenon often involves the entire pow er system. Indeed, the rotor angle of the machine is the main variable involved in a voltage collapse. For this reason, there is not difference between a voltage collapse, angle collapse and classical instability.

The main difference betw een a voltage collapse and classical transient stability is that the voltagecolapse focuses on loads and voltage magnitudes, w hereas classical transient stability focuses on generators and angles. In addition, voltage collapse often includes longer time scale dynamics as w ell as the effects of discrete events such as line outages. 
A voltage collapse can be seen as a saddle-node bifurcation of equilibrium points. A saddle-node bifurcation is a disappearance of equilibrium points as a parameter value changes slow ly. Thesaddle-node bifurcation of most interest occurs $w$ hen the equilibrium point where the pow er system operates disappears. As a saddle-node bifurcation can produce a voltage collapse, it is useful to study this phenomenon to understand and avoid it.

In this way, the work reported in this paper presents the elimination of a saddle-node bifurcation inabasic pow er system proposed in [6] using a classical PID controller. We found the conditions that the systemand the controller must satisfied such that this elimination can be achieved. Also, we analyze the stability of the rotor angle and its frequency, w hich are directly related to the voltage collapses. In this way one can expect that the results obtained here could be extended to more general systems displaying these undesirable phenomena.

\section{A POWER SYSTEM MODEL}

The equation that describes the rotor motion of a synchronous generator is

$$
\ddot{J} \dot{\theta}=T_{a} \quad N \cdot m
$$

w here $J$ is the equivalent inertial momentum of all the loads attached to the rotor, $\theta$ is the mechanicalangle of the shaft, measured $w$ ith respect to a static framew ork, and $T_{a}$ is the resultant torque driving the axis. The machine is a generator, so the driven torque $T_{m}$ is mechanical and the reacting torque is an electric torque, so that

$$
T_{a}=T_{m}-T_{e}
$$

A positive mechanical torque accelerates the rotor, while a positive reacting torque decelerates the machine. If $w$ e consider a synchronous rotating reference framew ork moving $w$ ith aconstantspeed $\omega_{R}$, the angle $\theta$ can be expressed as

$$
\theta=\left(\omega_{R} t+\alpha\right)+\delta_{m},
$$

where $\alpha$ is a constant and $\delta_{m}$ is the angular difference betw een the mechanical angle and the moving frame. Hence, equation (1) is transformed to

$$
J \dot{\delta}_{m}=J \cdot \dot{\omega}_{m}=T_{m}-T_{e}
$$

where $\omega_{R}$ is the derivative of $\delta_{m}$. By multiplying both sides of (2) by $\omega_{R}$ w e obtain a description in terms of pow er, that is

$$
\omega_{m} J \ddot{\delta}_{m}=\omega_{m} T_{a}-\omega_{m} T_{m}-\omega_{m} T_{e}=P_{m}-P_{e}
$$

w here $J \omega_{R}$, denoted by $M$, is called the inertia constant, and $P_{m}$ and $P_{e}$ are the mechanical and electrical pow er, respectively.

Let us consider a system composed by tw o simple generators connected as show $\mathrm{n}$ infigure(1), the power supplied by the source $E \angle \delta_{m}$ is given by 


$$
P_{e}=\frac{E V}{X} \sin \delta_{m}
$$

where $X$ is the load.

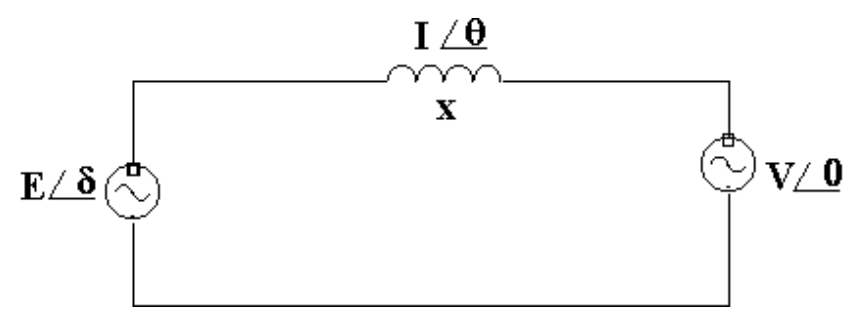

Figure 1. A basic power system.

If a dissipation component $D \omega$ inherent to the machine is added, then the model of this basic pow er system is given by

$$
\begin{aligned}
\dot{\delta} & =\omega \\
\dot{\omega} & =\frac{1}{M}\left[P_{m}-\frac{E V}{X} \sin (\delta)-D \omega\right]
\end{aligned}
$$

\section{A SADDLE-NODE BIFURCATION}

From equation (4), we see that the equilibrium point is given by

$$
\omega_{o}=0, \quad \delta_{o}=\arcsin \frac{P_{m} X}{E V}
$$

If we take as parameter the reactance $X$, it can seen directly from this expression that the system has a saddle-node bifurcation at the points $(X, \delta)=( \pm 1, \pm \pi / 2)$.

The Jacobian matrix of (4), evaluated at the equilibrium point (5) is

$$
A=\frac{\partial f}{\partial x}=\left[-\frac{E V}{M X} \cos \left(\arcsin \frac{P_{m} X}{E V}\right)-\frac{D}{M}\right]
$$

w hich has the eigenvalues

$$
\lambda_{1,2}=\frac{D}{M}\left(-1 \pm \sqrt{1-\frac{4 M E V}{D^{2} X} \sqrt{1-\left(\frac{P_{m} X}{E V}\right)^{2}}}\right)
$$

From Eq. (7) it can be seen that a zero eigenvalue exist $w$ hen $X= \pm E V / P_{m}$; these values are candidates to be bifurcation points.

The existence of the saddle-node bifurcation can be proved analytically with Sotomayor's theorem[8]. This theorem establishes the follow ing conditions to have this kind of bifurcation, 


$$
\begin{aligned}
& \omega^{T}\left(\frac{\partial f}{\partial \theta}\right) \neq 0, \\
& \omega^{T}\left[\left(\frac{\partial^{2} f}{\partial x^{2}}\right)_{0}(v, v)\right] \neq 0,
\end{aligned}
$$

where $v$ and $\omega$ are egenvectors corresponding to the zero eigenvalue of matrix $A$ (equation (6) and $A^{T}$, respectively), and the subindex 0 denotes the evaluation at the equilibrium point. They are given by

$$
v=\left(\begin{array}{l}
1 \\
0
\end{array}\right), \quad \omega=\left(\begin{array}{c}
1 \\
\frac{M}{D}
\end{array}\right),
$$

from w here

$$
\omega^{T}\left(\frac{\partial f}{\partial \theta}\right)=\frac{P_{m}^{2}}{V D} \neq 0
$$

and

$$
\omega^{T}\left[\left(\frac{\partial^{2} f}{\partial x^{2}}\right)_{0}(v, v)\right]=\frac{P}{D} \neq 0
$$

Therefore, the conditions established by Sotomayor's theorem are satisfied, and the system displays a saddle-node bifurcation at the point

$$
\left(\omega_{0}, \delta_{0}, X_{0}\right)=\left(0, \arcsin \left(\frac{P_{m} V}{E V}\right), \frac{V E}{P_{m}}\right)
$$

\section{CONTROL OF A SADDLE-NODE BIFURCATION USING A PID CONTROLLER}

Let us consider that the mechanical pow er is given by $P_{m}=P_{0}+v$, w here $P_{0}$ is a nominal input and $v$ is an adjustment $w$ ith control purposes that can be expressed as $v=M u$, therefore the system (4) transforms to

$$
\begin{aligned}
& \dot{\delta}_{m}=\omega \\
& \dot{\omega}=\frac{1}{M}\left[P_{0}-\frac{V}{X} \sin \delta_{m}-D \omega\right]+u
\end{aligned}
$$

The controller proposed has the structure show $\mathrm{n}$ in figure 2, where the input control $u$ is given by

$$
u=k_{p} e+k_{d} e+\int_{0}^{t} e d t
$$

where $e=\delta_{\text {ref }}-\delta_{m}$ is the error and $\delta_{\text {ref }}$ is a constant reference angle. 


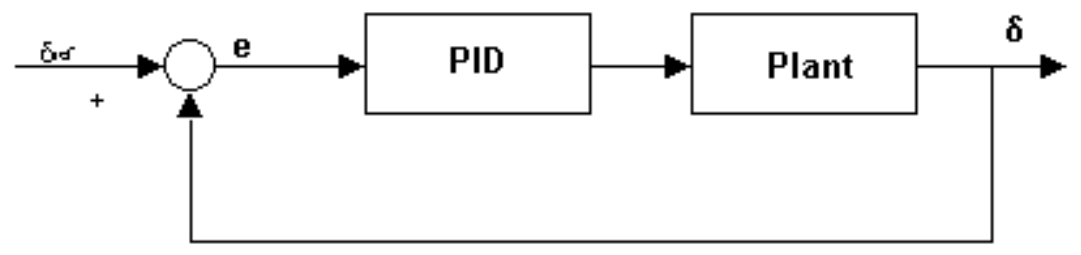

Figure 2. A block diagram of the closed-loop system.

The system (8) can be reduced to a differential equation given by

$$
\begin{aligned}
& \delta_{m}=\frac{1}{M}\left[P_{0}-\frac{V}{X} \sin \delta_{m}-D \omega\right]+u \\
& \cdots \delta_{m}=\frac{1}{M}\left[-\frac{V}{X} \delta_{m} \cos \delta_{m}-D \ddot{\delta_{m}}\right]-k_{p} \dot{\delta}_{m}-k_{d} \ddot{\delta}_{m}+k_{i}\left(\delta_{r e f}-\delta_{m}\right)
\end{aligned}
$$

Now, the system (11) has the new state space form given by

$$
\begin{aligned}
& \dot{x}_{1}=x_{2}, \\
& \dot{x}_{2}=x_{3}, \\
& \dot{x}_{3}=k_{i}\left(\delta_{r e f}-x_{1}\right)-\left(k_{p}+\frac{V}{M X} \cos x_{1}\right) x_{2}-\left(k_{d}+\frac{D}{M}\right) x_{3},
\end{aligned}
$$

where $x_{1}=\delta_{m} \quad x_{2}=\omega$ and $x_{3}=\omega$.

\subsection{Equilibrium points}

From equation (12) we see that the equilibrium points are given by

$$
x_{1}=\delta_{r e f}, x_{2}=0, x_{3}=0
$$

From this expression and using the implicit function theorem, it is possible to see that this is the only equilibrium point; therefore, a saddle-node bifurcation cannot be presented anymore.

\section{STABILITY ANALYSIS OF THE CLOSED-LOOP SYSTEM}

The Jacobian matrix of the system (12) is given by

$$
A=\left[\begin{array}{ccc}
0 & 1 & 0 \\
0 & 0 & 1 \\
-k_{i}+\frac{V}{M X} \sin \left(x_{1}\right) x_{2} & -\left[k_{p}+\frac{V}{M X} \cos x_{1}\right] & -\left[k_{d}+\frac{D}{M}\right]
\end{array}\right]
$$

w hich, evaluated at equilibrium point $x_{1}=\delta_{\text {ref }}, x_{2}=0, x_{3}=0$, results in 


$$
A=\left[\begin{array}{ccc}
0 & 1 & 0 \\
0 & 0 & 1 \\
-k_{i} & -\left[k_{p}+\frac{V}{M X} \cos \delta_{r e f}\right] & -\left[k_{d}+\frac{D}{M}\right]
\end{array}\right]
$$

This matrix has the characteristic polynomial

$$
Z^{3}+\left(k_{d}+\frac{D}{M}\right) Z^{2}+\left(k_{p}+\frac{V}{M X} \cos \delta_{r e f}\right) Z+k_{i}=0 .
$$

Let us consider a numerical example given by the values $V=1, D=M=0.5$ proposed in [6], and $k_{p}=k_{d}=k_{i}=1$. This leads to the characteristic polynomial

$$
Z^{3}+2 Z^{2}+\left(1+\frac{2}{X} \cos \delta_{r e f}\right) Z+1=0
$$

Using the Routh-Hurw itz criteria and, considering that $\delta_{\text {ref }}$ is limited to $0 \leq \delta_{\text {ref }} \leq \pi / 2$, the follow ing stability conditions are obtained

$$
\begin{aligned}
& \left(1+\frac{2}{X} \cos \delta_{r e f}\right)>0 \Rightarrow X \neq 0 \& X>-2, \\
& \frac{2}{X} \cos \delta_{r e f}+0.5>0 \Rightarrow X \neq 0 \& X>-4 .
\end{aligned}
$$

Because $X$ is positive, then the conditions (13) and (14) are alw ays satisfied by the closed-loop system Figures (3) and (4) show the behavior of the last tw o expressions, show ing that the stability of theclosedloop system does not depend on the parameter values.

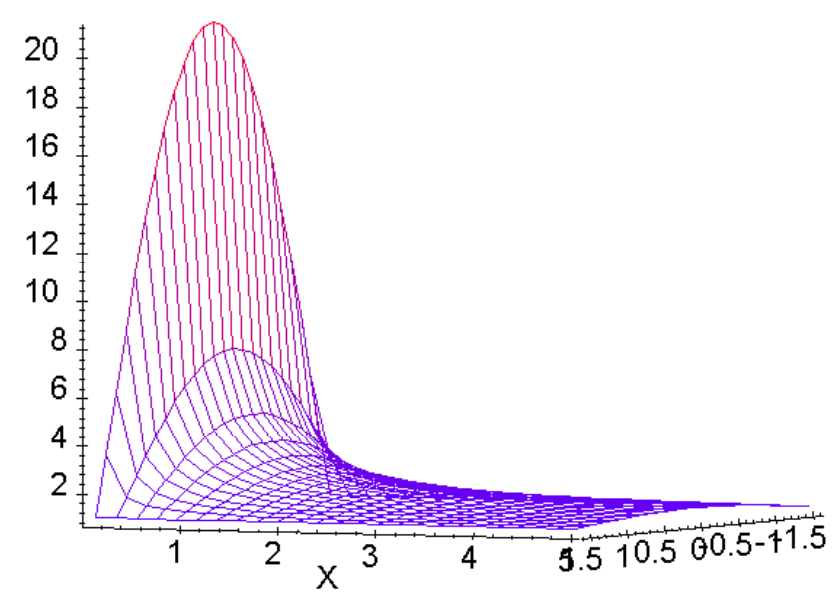

Figure 3. Graph of $f=1+2 / X \cos \delta_{\text {ref }}$ (condition (13)). 


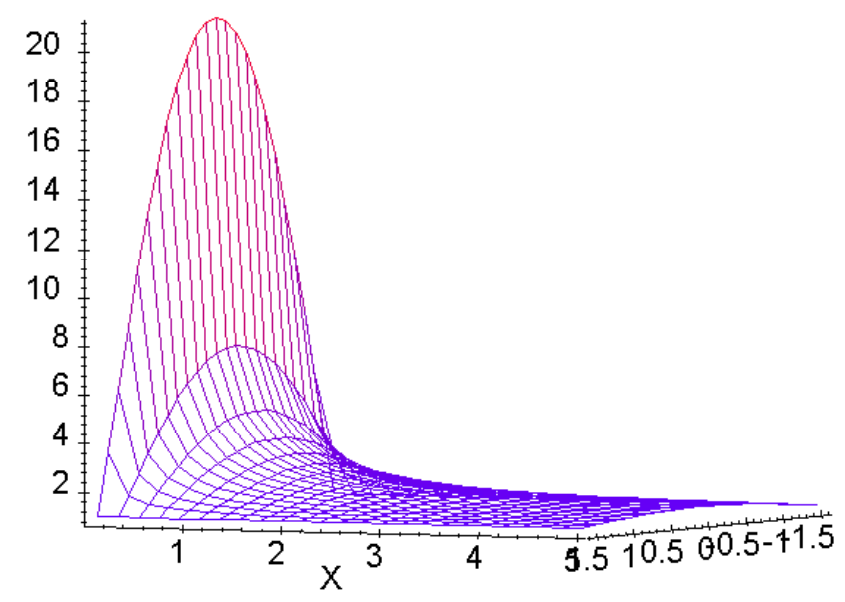

Figure 4. Graph of $f=0.5+2 / X \cos \delta_{\text {ref }}($ condition (14)).

Figures (5) and (6) show tw o numerical simulations, for $\delta_{\text {ref }}=0$ and $\delta_{\text {ref }}=\pi / 2$. We can see that the angle $\delta_{m}$ follow s the reference $\delta_{\text {ref }}$ and $\omega \rightarrow 0$ as $t \rightarrow \infty$.

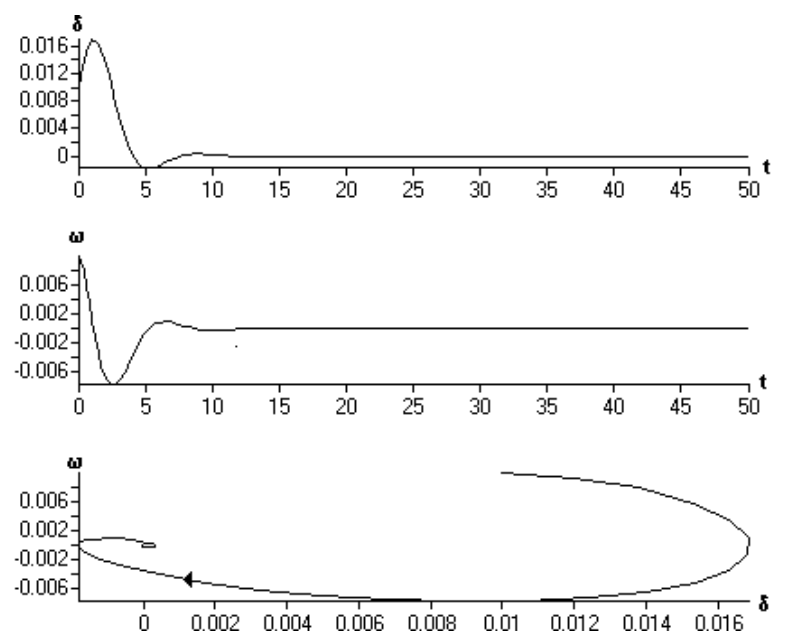

Figure 5. Response of the controlled system for $\delta_{\text {ref }}=0$ and $X=2$.
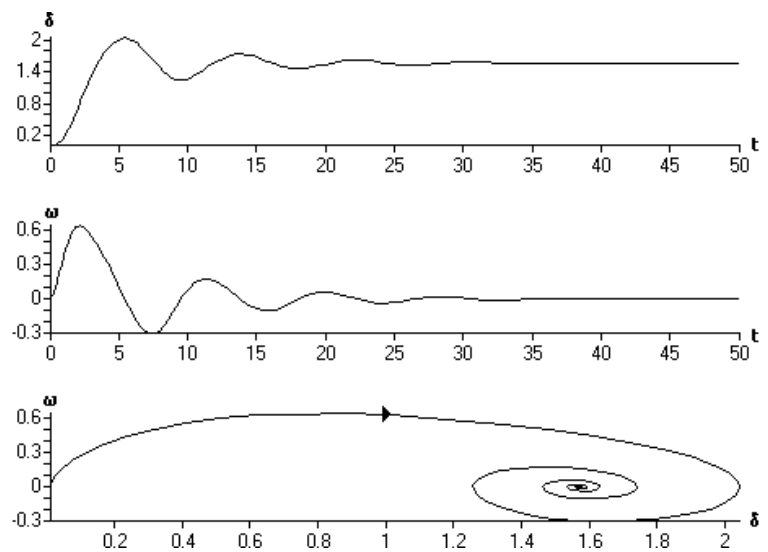

Figure 6. Response of the controlled system for $\delta_{\text {ref }}=\pi / 2$ and $X=2$. 


\section{CONCLUSIONS}

In this paper we have analytically proved the existence of saddle-node bifurcations in a simplified model of a pow er system. This dynamical phenomenon is related to voltage collapses.

We have also proved that the introduction of a simple classical PID controller can eliminate this type of bifurcation, eliminating at least partially the possibility of having collapses in the system.

A power system is very complicated, so we can expect that a real process $w$ ill present many more complex phenomena, as is indeed the case. The preliminary analysis presented here could be useful, how ever, to define a systematic $w$ ay to analyze the conditions to have dangerous behaviors of these important processes, and to envisage some possible form to avoid, or at least to delay these high-risk situations.

\section{REFERENCES}

[1] Wang, HO, Abed, EH, 1993 Control of nonlinear phenomena at the inception of voltage collapse, Internal Report. Institute for Systems Research.

[2] Abed E H., Hamdan A. M., Alexander J. C. 1993 Dynamical bifurcations in pow er system exhibing a voltage collapse, International Journal on Bifurcation and Chaos, Vol 3, No. 5, pp. 1169-1174.

[3] Dobson I., Liming Lu. 1993 New methods for computing a closest saddle-node bifurcation and w orst case load pow er margin for voltage collapse, IEEE Transaction on Power Systems, Vol8, pp 905-913.

[4] Dobson I., Chiang D., Thorp J. S., 1988 A model of voltage collapse in electric pow er systems, Proceedings of the 27th Conference on Decision and Control, Austin, Texas, December.

[5] Dobson I. and Chiang D., 1989Tow ards theory of voltage collapse in electric pow er systems, Systems \& Control Letters, Vol.13, pp. 253-262.

[6] Cañizares C. A., et al. 1995 On bifurcations, voltage collapse and load modeling, IEEE Transaction on power systems. Vol. 10, No. 1, pp. 512-522, February.

[7] Cañizares C. A., 1999 Hopf bifurcation control in pow er systems using pow er system stabilizers and static VAR compensators, North American Power Symposium, San Luis Obispo, California, Octubre.

[8] Perco L. 1996, Differential equations and dynamical systems, Spring Verlag.

[9] Srivastava K. N. and Srivastava S. C.. 1998 Elimination of dynamical bifurcation and chaos in pow er systems using facts devices, IEEE Transactions on Circuits and Systems, Fundamental Theory and Applications, Vol. 45, No. 1, January. 
Authors Biography

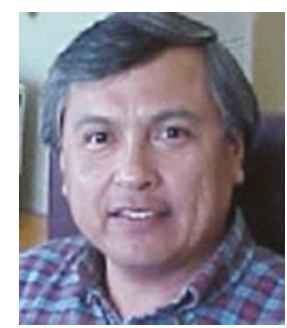

Joaquín Alvarez Gallegos

Is an electronics and telecommunications engineer from ESIME (IPN, 1975), master of sciences in electrical engineering from CINVESTAV (1976), and PhD from INGP (Grenoble, France, 1979). He has been a researcher at CINVESTAV and the Electrical Research Institute, and professor of ESIME (IPN) and CENIDET. Currently he is working at the Electronics and Telecommunications Department of CICESE (Scientific Research and Advances Studies Center of Ensenada, BC, Mexico). His main research interests are in nonlinear control and the analysis, synchronization, and control of chaotic systems. Dr. Alvarez is a member of the National System of Researchers (SNI), the Mexican Academy of Sciences (AMC), the National Academy of Engineering (ANI), and the Mexican Association of Automatic Control (AMCA).

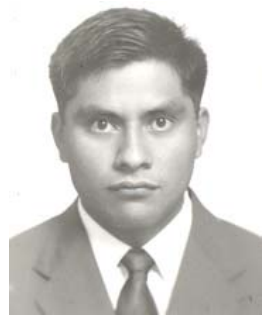

\section{David I. Rosas Almeida}

Was born in Tlaixpan, Mexico (1975); he is an Electronics Engineer from BUAP (1997), and received the master's degree in electronics and telecommunications from CICESE in 1999. Since 1999 he has been a professor in the UABC. His main research interests are analysis and control of bifurcations, nonlinear control, and synchronization of nonlinear dynamical systems.

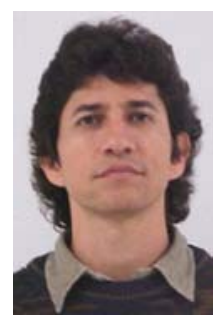

\section{César Amaro Hernández}

Was born in Mexicali, Baja California, México, in 1965. He received the electrical engineer (1987) and the master in engineering degrees from the Universidad Autónoma de Baja California. Since 1989 he has been a professor in the Engineering Faculty at the Universidad Autónoma de Baja California, where currently he is in charge of the Electrical Engineering Department. His areas of interest are electric circuit analysis, power systems and control systems. 